\title{
WAVE INTENSITIES AND SLOPES IN LAGRANGIAN SEAS
}

\author{
SOFIA ÅBERG, ${ }^{*}$ Lund University
}

\begin{abstract}
In many applications, such as remote sensing or wave slamming on ships and offshore structures, it is important to have a good model for wave slope. Today, most models are based on the assumption that the sea surface is well described by a Gaussian random field. However, since the Gaussian model does not capture several important features of real ocean waves, e.g. the asymmetry of crests and troughs, it may lead to unconservative safety estimates. An alternative is to use a stochastic Lagrangian wave model. Few studies have been carried out on the Lagrangian model; in particular, very little is known about its probabilistic properties. Therefore, in this paper we derive expressions for the level-crossing intensity of the Lagrangian sea surface, which has the interpretation of wave intensity, as well as the distribution of the wave slope at an arbitrary crossing. These results are then compared to the corresponding intensity and distribution of slope for the Gaussian model.
\end{abstract}

Keywords: Crossing theory; wave steepness; Gaussian process; Palm distribution; Rice's formula

2000 Mathematics Subject Classification: Primary 60G60

Secondary 60G15; 60K40; 86A05

\section{Introduction}

Stochastic wave models are derived as approximations from general ocean surface-wave theory. The simplest approximation based on the Eulerian equations of motion, i.e. the conservation laws written for a still volume of fluid, leads to the Gaussian or linear wave model. This model has been successfully used in ocean engineering since the 1950s; see, e.g. [15] and [11]. The main advantage of the Gaussian model is its simplicity-the correlation structure or, equivalently, the frequency spectrum, commonly used in marine sciences, determines all stochastic properties of the waves. Moreover, the theory of Gaussian processes is well known so that, for example, statistical distributions of different wave characteristics such as wave period, crest amplitude, and steepness can be studied in detail; see, e.g. [1], [2], [3], and [13]. However, the simplicity of the Gaussian model is also one of its drawbacks, in the sense that it is too simple to catch important features of real ocean waves. Ocean waves are typically asymmetric, as opposed to the waves generated by the Gaussian model. By asymmetry it is either meant crest-trough asymmetry, meaning that the crests are peaked and narrow, and the troughs wide and shallow, or front-back asymmetry, which refers to the fact that the wave fronts are usually steeper than the wave rears. The symmetry, or rather the lack of asymmetry, of Gaussian waves is particularly unfavorable when we want to describe quantities such as wave steepness, which, for example, is important for safety calculations of offshore structures.

Received 13 December 2006; revision received 18 June 2007.

* Current address: Matematiska vetenskaper, Chalmers tekniska högskola, Göteborg SE-412 96, Sweden.

Email address: abergs@chalmers.se 
Another class of physically based wave models are the Lagrangian models, whose origin dates back to the work of Lagrange [7]. These models consider not only vertical but also horizontal motions of the individual fluid particles and, in the stochastic formulation, these motions are modeled by Gaussian processes. As a result, the surface elevation is described through a parametric representation which allows for a larger flexibility in the wave shape. These models have received much less attention in the literature than the Gaussian models. However, recently several authors [5], [6], [14] have noted that a stochastic Lagrangian model can produce crest-trough asymmetry as well as front-back asymmetry, the latter, in particular, produced for higher-order Lagrangian models. Thus, the Lagrangian model produces asymmetric waves at the same time as it retains enough of the Gaussian structure to allow the vast theory on Gaussian processes to be used in the calculations.

There are very few studies of the probabilistic properties of the stochastic Lagrangian wave models. Recently, however, Lindgren [9] derived the so-called Slepian models for Lagrangian seas. In this study we take a somewhat different approach and our first goal is to derive crossing intensities for the Lagrangian model, i.e. to derive how many times per time or space unit the sea surface crosses a certain level. Since, in the oceanographic community, a 'wave' is often defined as the part of the sea surface between two successive upcrossings of the still water level, these crossing intensities can also be interpreted as wave intensities. Our second goal is to derive the Palm distributions of slopes (wave steepness) for two particular cases. The first case deals with finding the distribution of wave steepness for so-called space waves, by which we mean that the sea surface at a fixed time point is considered. The second case considers wave steepness of the surface elevation at a fixed location, referred to as a time wave. Important areas of application, motivating this study, are remote sensing, where good models for wave steepness are crucial when estimating back-scattered radar, and marine safety, as steep waves can cause significant slamming loads on ships and offshore structures.

With a Gaussian model the slope at an arbitrary crossing can be found to have a Rayleigh distribution, for both the time and space waves. Moreover, this distribution will not depend on what level is being crossed. However, in this paper we will show that this is not the case for the Lagrangian model, where the distribution of slope typically depends on the crossing level. In particular, we will see that the Lagrangian wave is steeper than a Gaussian wave if positive crossing levels are considered, whereas the opposite is true for negative crossing levels.

The organization of this paper is as follows. First the stochastic Gaussian and Lagrangian wave models are presented. We then define the Palm distributions, since these are the kind of distributions that we will later apply to finding the distribution of slope at an arbitrary crossing. After these introductory sections, the crossing intensity and the distribution of slope for Lagrange space waves are considered. The results from the computations in Section 4 are then exemplified and compared to the corresponding results for a Gaussian wave model. In Section 5 expressions for the wave intensity and the distribution of slope for Lagrange time waves are derived and exemplified.

\section{Gaussian and Lagrangian wave models}

In this paper two different wave models will be considered, namely, stochastic versions of Eulerian and Lagrangian wave models of the first order. Both these models, representing the two main approaches in fluid mechanics for describing the motion of a fluid, are physically based and are obtained by solving certain hydrodynamical equations with suitable boundary conditions. 
The stochastic Eulerian wave model, known as the Gaussian wave model, describes the sea elevation $W(t, x)$ by means of a zero-mean stationary Gaussian process. Using the spectral representation for stationary processes (see, e.g. [4, pp. 128-135]) the sea elevation can be written as

$$
W(t, x)=\int_{-\infty}^{\infty} \mathrm{e}^{\mathrm{i}(\kappa(\omega) x-\omega t)} \mathrm{d} \xi(\omega) .
$$

Here $\xi(\omega)$ is a complex spectral process having zero mean and independent Gaussian increments satisfying $\mathrm{d} \xi(-\omega)=\overline{\mathrm{d} \xi(\omega)}$, which ensure that the process $W(t, x)$ is real valued. The wave number $\kappa$ in (1) is a function of the frequency $\omega$. This is due to the dispersion relation which is a consequence of the boundary conditions and the governing equations of the fluid. Letting $h$ denote the water depth and $g$ the gravitational acceleration, the dispersion relation is given by

$$
\omega^{2}=g|\kappa| \tanh (|\kappa| h) .
$$

If $\kappa$ and $\omega$ are chosen to have the same sign then the waves will travel in the direction of the positive $x$-axis and if they have opposite signs then the converse will be true; see also Appendix A.

In the Gaussian model the surface elevation $W(t, x)$, i.e. the vertical movement of the sea surface, is given for each fixed point $x$. The Lagrangian model uses a different approach. In this model the sea is described through a set of water particles, each labeled by a reference coordinate, $u$ say, representing the particle's location at rest. At a given time $t$, the particle with reference coordinate $u$ is located at $(X(t, u), W(t, u))$, where $X(t, u)$ gives the horizontal location and $W(t, u)$ the vertical location with respect to some Cartesian coordinate system having its origin at the still water level. Thus, the Lagrangian approach models both vertical and horizontal movements, as opposed to the Gaussian model. In a stochastic formulation, as defined by [9], the processes $X$ and $W$ are modeled by Gaussian processes. In particular, the $X$-process can be written as a linear filtering of the $W$-process in the following fashion:

$$
\begin{gathered}
W(t, u)=\int_{-\infty}^{\infty} \mathrm{e}^{\mathrm{i}(\kappa(\omega) u-\omega t)} \mathrm{d} \xi(\omega), \\
X(t, u)=u+\int_{-\infty}^{\infty} \mathrm{i} \frac{\cosh (\kappa(\omega) h)}{\sinh (\kappa(\omega) h)} \mathrm{e}^{\mathrm{i}(\kappa(\omega) u-\omega t)} \mathrm{d} \xi(\omega) .
\end{gathered}
$$

In the following, we will always assume that the processes $W(t, u)$ and $X(t, u)$ have continuously differentiable sample paths and the derivatives will be denoted by a subscript, e.g. $W_{t}(t, u)$ denotes the derivative of $W(t, u)$ with respect to time. Sometimes the Lagrange wave height at a fixed location $x$ at a given time $t$ will be denoted by $L(t, x)$. Using the $X$-process and $W$-process we may thus write $L(t, X(t, u))=W(t, u)$.

One problem with the Lagrangian model, without any reasonable physical interpretation, is that the sea surface may 'fold'. Folding occurs if more than one particle happens to be at the same location $x_{0}$ at a certain time $t_{0}$, i.e. if the equation $X\left(t_{0}, u\right)=x_{0}$ has more than one solution. If this is the case then the Lagrange wave height, $L\left(t_{0}, x\right)$, is not unique but takes several values. As noted in [9], the probability of folding is low, especially for deep water.

\section{Palm distributions}

Let $t$ be a one-dimensional parameter, which may represent either time or space, and denote by $N_{v}^{Z}([0, T])$ the number of times a stationary process $Z(t)$ takes the value $v$ in the interval 
$[0, T]$. Furthermore, let $N_{v}^{Z}([0, T], A)$ be the number of times a stationary process takes the value $v$ and, at the same time, satisfies a statement $A$ about the process, its derivatives, or another process. Here $A$ could, for instance, be the statement ' $Z$ ' $(t) \leq z$ ', where $Z^{\prime}(t)$ denotes the derivative of $Z(t)$ with respect to time. A so-called Palm measure is defined by the following ratio of intensities:

$$
\mathrm{P}_{v}^{Z}(A)=\frac{\mathrm{E}\left[N_{v}^{Z}([0,1], A)\right]}{\mathrm{E}\left[N_{v}^{Z}([0,1])\right]}
$$

provided that the expected number of $u$-crossings, $\mathrm{E}\left[N_{v}^{Z}([0,1])\right]$, is finite. If the process $Z(t)$ is ergodic then the intensities in (2) can be computed as sample averages and, with probability 1 ,

$$
\mathrm{P}_{v}^{Z}(A)=\lim _{T \rightarrow \infty} \frac{N_{v}^{Z}([0, T], A)}{N_{v}^{Z}([0, T])} .
$$

Thus, the Palm distribution has the empirical interpretation that it is the long-term proportion of $v$-crossings by the process $Z$ for which $A$ is satisfied. Note that a Gaussian process is ergodic if and only if its spectral distribution is continuous; see [4, p. 157].

In Lemma 1, below, a stationary Gaussian vector-valued process $\left(Z(t), Z^{\prime}(t), Y(t)\right)$ will be considered. The process $Y(t)$ can either be directly related to $Z$, e.g. we may have $Y(t)=Z^{\prime \prime}(t)$, or it can be any other process correlated with $Z(t)$. In order to simplify the notation we introduce the random variables $Y_{v}$ and $Z_{v}^{\prime}$ having joint distribution function $F_{Y_{v}, Z_{v}^{\prime}}(y, z)$ defined by the Palm measure, (2), i.e.

$$
F_{Y_{v}, Z_{v}^{\prime}}(y, z)=\mathrm{P}\left(Y_{v} \leq y, Z_{v}^{\prime} \leq z\right)=\mathrm{P}_{v}^{Z}\left(Y(t) \leq y, Z^{\prime}(t) \leq z\right) .
$$

These random variables are a special case of the so-called Slepian model process (see [10] for a detailed presentation) and we may think of them as $Y(t)$ and $Z^{\prime}(t)$ observed at a randomly chosen $v$-crossing of the process $Z(t)$. Lemma 2, below, which will prove useful in the analysis of space waves, gives an explicit representation of these variables.

Lemma 1. Suppose that $\left(Z(t), Z^{\prime}(t), Y(t)\right)$ is a stationary Gaussian vector-valued process such that the density of $\left(Z(t), Z^{\prime}(t), Y(t)\right)$ exists. Furthermore, assume that the sample paths of $Y(t)$ and $Z(t)$ are almost surely (a.s.) continuous and a.s. continuously differentiable, respectively. Let $m_{Z}=\mathrm{E}(Z(t))$ and $m_{Y}=\mathrm{E}(Y(t))$, and let the covariance matrix $\Sigma$ of the vector $\left(Z(t), Z^{\prime}(t), Y(t)\right)$ be given by

$$
\boldsymbol{\Sigma}=\left(\begin{array}{ccc}
r^{z z} & 0 & r^{z y} \\
0 & r_{t t}^{z z} & r_{t 0}^{z y} \\
r^{z y} & r_{t 0}^{z y} & r^{y y}
\end{array}\right),
$$

where the superscripts indicate what processes are involved and the subscripts indicate with respect to which variable the processes are differentiated. Then the variables $Y_{v}$ and $Z_{v}^{\prime}$, with distribution defined by (3), have the following representations:

$$
Z_{v}^{\prime} \stackrel{\mathrm{D}}{=} \sqrt{r_{t t}^{z z}} R \quad \text { and } \quad Y_{v} \stackrel{\mathrm{D}}{=} m_{v}+\frac{r_{t 0}^{z y}}{\sqrt{r_{t t}^{z z}}} R+\sqrt{r^{y y}-\frac{\left(r^{z y}\right)^{2}}{r^{z z}}-\frac{\left(r_{t 0}^{z y}\right)^{2}}{r_{t t}^{z z}}} U
$$

where $\stackrel{\mathrm{D}}{=}$ denotes equality in distribution, $U$ is the standard normal, $R$ is independent of $U$, with a double Rayleigh distribution, i.e. with probability density $f(r)=(|r| / 2) \mathrm{e}^{-r^{2} / 2}$, and $m_{v}=m_{Y}+\left(r^{z y} / r^{z z}\right)\left(v-m_{Z}\right)$. 
Proof. The proof is straightforward using the definition of the Palm probability, Rice's formula for marked crossings (see, e.g. [8, Lemma 7.5.2]), and the Gaussian regression formulae.

Remark 1. The Palm measure, (2), is defined for $v$-crossings of the process $Z$. In an analogous fashion, Palm measures at $v$-upcrossings may be defined. In this case Lemma 1 is still valid with $R$ being a Rayleigh variable having density $f(r)=r \mathrm{e}^{-r^{2} / 2}, r>0$.

\section{Lagrange space waves}

In this section the Lagrangian sea surface is considered at a fixed time point, i.e. as seen on a photo or from a satellite. Our primary goal is to find the crossing intensities (wave intensities) and the Palm distributions of the spatial derivative at the crossing and a secondary goal is to compare these to what is obtained from the Gaussian model.

Let $t_{0}$ be a fixed time point and $L\left(t_{0}, x\right)$ be the surface elevation at location $x$ at time $t_{0}$ according to the Lagrangian model. We will refer to $L\left(t_{0}, x\right)$ as the Lagrange space wave and owing to the definition of the Lagrangian sea it satisfies $L\left(t_{0}, X\left(t_{0}, u\right)\right)=W\left(t_{0}, u\right)$. By differentiating this relation with respect to $u$, the following formula for the slope of the Lagrange space wave is obtained:

$$
L_{x}\left(t_{0}, X\left(t_{0}, u\right)\right)=\frac{W_{u}\left(t_{0}, u\right)}{X_{u}\left(t_{0}, u\right)} .
$$

Note that, due to folding, $L_{x}\left(t_{0}, x\right)$ may not be uniquely defined for a specific $x$-value. However, by working with the underlying processes $W\left(t_{0}, u\right)$ and $X\left(t_{0}, u\right)$ rather than $L\left(t_{0}, x\right)$ itself, the folding problem can be reduced and (4) can be used to define the derivative at crossings.

Theorem 1, below, gives the crossing intensity and the Palm distribution of slope for Lagrange space waves. Its result is far from surprising since the Palm distribution for the Lagrange space wave turns out to be the Palm distribution of $W_{u}\left(t_{0}, u\right) / X_{u}\left(t_{0}, u\right)$ in the Gaussian model.

Theorem 1. (Space waves.) (a) The v-crossing intensity of the Lagrange space wave, $L\left(t_{0}, x\right)$, equals the $v$-crossing intensity of the Gaussian process $W\left(t_{0}, u\right)$, i.e.

$$
\mathrm{E}\left[N_{v}^{L}([0,1])\right]=\mathrm{E}\left[N_{v}^{W}([0,1])\right]=\frac{1}{\pi} \sqrt{\frac{r_{u u}^{w w}}{r^{w w}}} \mathrm{e}^{-v^{2} / 2 r^{w w}} .
$$

(b) The slope observed at an arbitrary $v$-crossing $x_{k}$ of $L\left(t_{0}, x\right)$ has the following distributional representation:

$$
L_{x}\left(t_{0}, x_{k}\right) \stackrel{\mathrm{D}}{=} \frac{R \sqrt{r_{u u}^{w w}}}{1+\left(r_{0 u}^{w x} / r^{w w}\right) v+U \sqrt{r_{u u}^{x x}-\left(r_{0 u}^{w x}\right)^{2} / r^{w w}}},
$$

where $R$ and $U$ are independent double Rayleigh $\left(f_{R}(r)=(|r| / 2) \mathrm{e}^{-r^{2} / 2}\right)$ and standard normal variables, respectively. If $x_{k}$, instead, is an arbitrary crossing corresponding to an upcrossing $u_{k}$ of the process $W\left(t_{0}, u\right)$ then the result is still valid replacing $R$ with a Rayleigh variable having density $f_{R}(r)=r \mathrm{e}^{-r^{2} / 2}, r>0$.

Proof. The number of $v$-crossings of the Lagrange space wave having slope less than or equal to $y$ can be expressed as

$$
N_{v}^{L}\left([0,1], L_{x}\left(t_{0}, x\right) \leq y\right)=\#\left\{u \in \mathbb{R} ; W\left(t_{0}, u\right)=v, X\left(t_{0}, u\right) \in[0,1], \frac{W_{u}\left(t_{0}, u\right)}{X_{u}\left(t_{0}, u\right)} \leq y\right\} .
$$


By Rice's formula for marked crossings (see, e.g. [8, pp. 156-160]), it holds that

$$
\begin{aligned}
& \mathrm{E}\left[N_{v}^{L}\left([0,1], L_{x}\left(t_{0}, x\right) \leq y\right)\right] \\
& \quad=\int_{-\infty}^{\infty} \mathrm{E}\left[\left|W_{u}\left(t_{0}, u\right)\right| \mathbf{1}_{\left\{X\left(t_{0}, u\right) \in[0,1], W_{u}\left(t_{0}, u\right) / X_{u}\left(t_{0}, u\right) \leq y\right\}} \mid W\left(t_{0}, u\right)=v\right] f_{W\left(t_{0}, u\right)}(v) \mathrm{d} u
\end{aligned}
$$

By conditioning on $X_{u}\left(t_{0}, u\right)=w$, changing the order of integration, and making use of the dependence structure of the variables involved (see Appendix C), we find that

$$
\begin{aligned}
\mathrm{E}\left[N_{v}^{L}(\right. & {\left.\left.[0,1], L_{x}\left(t_{0}, x\right) \leq y\right)\right] } \\
= & \int_{0}^{\infty} f_{W\left(t_{0}, u\right), X_{u}\left(t_{0}, u\right)}(v, w) \int_{-\infty}^{w y}|z|\left\{\int_{0}^{1} \int_{-\infty}^{\infty} f_{W_{u}\left(t_{0}, u\right), X\left(t_{0}, u\right)}(z, x) \mathrm{d} u \mathrm{~d} x\right\} \mathrm{d} z \mathrm{~d} w \\
& +\int_{-\infty}^{0} f_{W\left(t_{0}, u\right), X_{u}\left(t_{0}, u\right)}(v, w) \int_{w y}^{\infty}|z|\left\{\int_{0}^{1} \int_{-\infty}^{\infty} f_{W_{u}\left(t_{0}, u\right), X\left(t_{0}, u\right)}(z, x) \mathrm{d} u \mathrm{~d} x\right\} \mathrm{d} z \mathrm{~d} w .
\end{aligned}
$$

By straightforward calculations, the innermost integrals (i.e. the terms in the curly brackets) can be shown to equal $f_{W_{u}\left(t_{0}, u\right)}(z)$. Using this result and rewriting (6) in terms of an expectation we obtain

$$
\mathrm{E}\left[N_{v}^{L}\left([0,1], L_{x}\left(t_{0}, x\right) \leq y\right)\right]=\mathrm{E}\left[N_{v}^{W}\left([0,1], \frac{W_{u}\left(t_{0}, u\right)}{X_{u}\left(t_{0}, u\right)} \leq y\right)\right] .
$$

Part (a) now follows by letting $y$ tend to $\infty$ in (7) and using Rice's formula for a stationary Gaussian process; see [4, pp. 193-200].

As (7) implies that

$$
\mathrm{P}_{v}^{L}\left(L_{x}\left(t_{0}, x\right) \leq y\right)=\mathrm{P}_{v}^{W}\left(\frac{W_{u}\left(t_{0}, u\right)}{X_{u}\left(t_{0}, u\right)} \leq y\right)
$$

(cf. (2)), part (b) follows by an application of Lemma 1 on the vector-valued process $\left(W\left(t_{0}, u\right)\right.$, $\left.W_{u}\left(t_{0}, u\right), X_{u}\left(t_{0}, u\right)\right)$, taking the covariances given in Appendix $\mathrm{C}$ into account.

Remark 2. In a Gaussian sea the distribution of slope at an arbitrary crossing is, according to Lemma 1, given by a Rayleigh variable and is not a function of the height of the crossing, i.e. it is independent of the value of $v$. As can be seen from (5), this is not the case for the Lagrangian sea.

By identifying the constants $a$ and $b$ in the following lemma, which can be proved by straightforward calculations, a closed-form expression for the density of the slope (at upcrossings) having representation (5) is obtained.

Lemma 2. Let $R$ be a Rayleigh variable with density $f_{R}(r)=r \mathrm{e}^{-r^{2} / 2}, r>0$, and let $U$ be a standard normal variable, independent of $R$. Furthermore, define $X=a R /(1+b U)$, where 

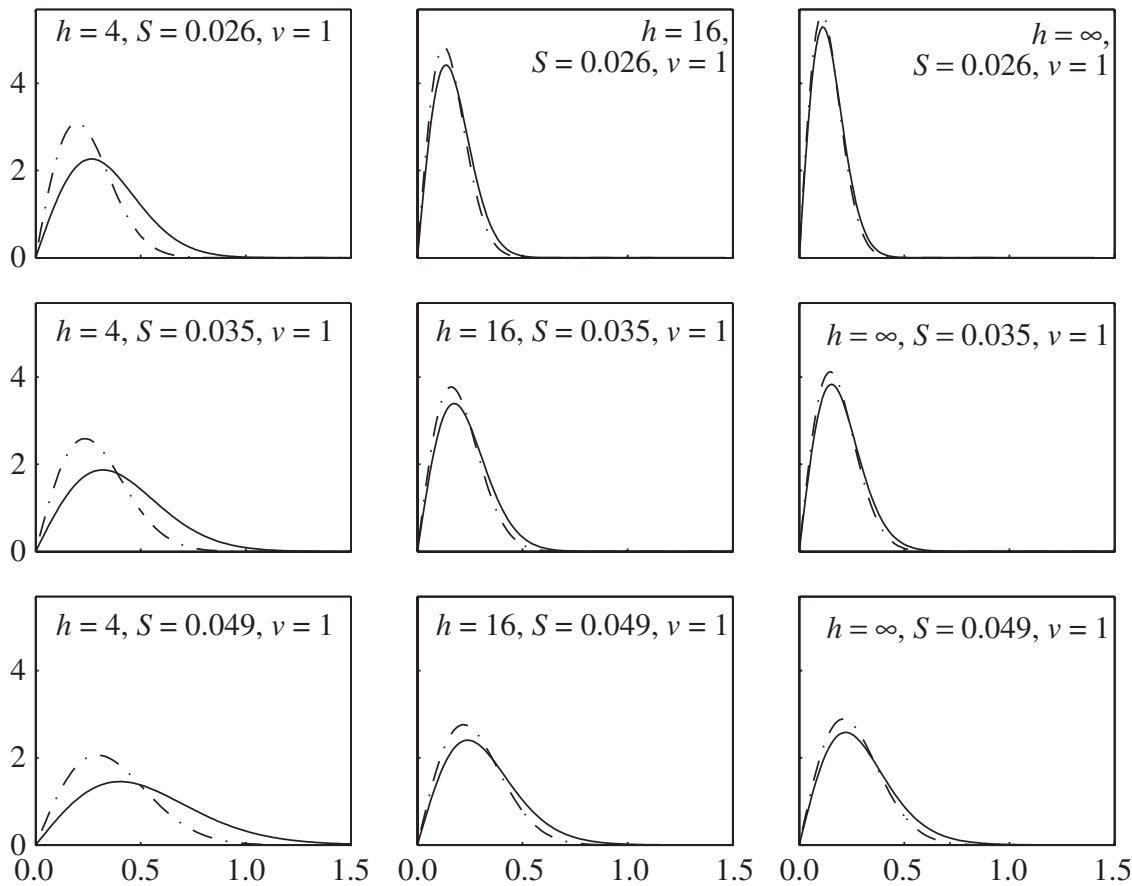

FIGURE 1: Density of slope observed at an arbitrary space-wave upcrossing of level $v=1 \mathrm{~m}$ in the Gaussian (dash-dotted line) and Lagrangian (solid line) models for different water depths, $h=4,16$, and $\infty \mathrm{m}$, and spectrum steepnesses, $S=0.026,0.035$, and 0.049 .

$a, b>0$. Then

$$
f_{X}(x)=\frac{\operatorname{sgn}(x) a}{\sigma^{3}(x)}\left(\frac{a^{2}}{\sigma^{2}(x)}+b^{2}\right) x \mathrm{e}^{-x^{2} / 2 \sigma^{2}(x)} \Phi\left(\frac{\operatorname{sgn}(x) a}{b \sigma(x)}\right)+\frac{1}{\sqrt{2 \pi}} \frac{a^{2} b}{\sigma^{4}(x)} x \mathrm{e}^{-1 / 2 b^{2}},
$$

where $\sigma(x)=\sqrt{a^{2}+b^{2} x^{2}}$ and $\Phi$ denotes the distribution function of a standard normal variable.

Having derived an expression for the Palm distribution of slope at space-wave crossings we are now ready to compare it to the distribution obtained by a Gaussian model.

Example 1. In this example the densities of slope at an arbitrary upcrossing in the Gaussian and Lagrangian models are compared. The models are defined by a frequency spectrum, in this case a spectra of the so-called Pierson-Moskowitz type; see Appendix B for details. This family of spectra is defined by two parameters, namely, the significant wave height $H_{\mathrm{s}}$ and the peak period $T_{\mathrm{p}}$. In our case we let $H_{\mathrm{s}}=7 \mathrm{~m}$ and we consider three different water depths, 4,16 , and $\infty \mathrm{m}$, and three different values of the steepness of the sea state, defined as $S=2 \pi H_{\mathrm{s}} / g T_{\mathrm{p}}^{2}$, namely, 0.026, 0.035, and 0.049. These different values represent moderately steep, steep, and very steep waves, respectively. We also choose a cut-off frequency $\omega_{\mathrm{c}}=33 / T_{\mathrm{p}} \mathrm{rad} \mathrm{s}^{-1}$, i.e. a frequency such that the spectrum is zero for frequencies higher than $\omega_{\mathrm{c}}$. This particular choice of parameters is for illustration purposes only, since in a real world situation it is unrealistic to have the same spectrum for different water depths. In Figure 1 the densities of slope are 
shown for the crossing level $v=1 \mathrm{~m}$ and it can be clearly seen that the Lagrangian model gives steeper waves than the Gaussian model. On the one hand, for a fixed steepness, the difference is greatest for shallow water and decreases with water depth. On the other hand, for fixed depth, the difference increases with steepness $S$. Thus, the densities resulting from the Lagrangian and Gaussian models differ most for steep sea states in shallow waters. For a negative crossing level the Lagrangian model will give less steep waves than the Gaussian model, whereas for zero-upcrossings very little difference can be found between the two models.

\section{Lagrange time waves}

Next we consider the Lagrangian sea surface at a fixed location, i.e. at a so-called Lagrange time wave, which might be appropriate in applications dealing with wave slamming on offshore structures. As for the space waves, the goal is to obtain expressions for the crossing intensities and the Palm distribution of the temporal derivative at the crossings. As we will see, the time waves are more difficult to analyze, mainly owing to the fact that we need to keep track of which particle $u$ is currently located at the fixed position.

Let $x_{0}$ be a fixed point in space and let $L\left(t, x_{0}\right)$ denote the Lagrange time wave, i.e. the sea elevation at location $x_{0}$ at time $t$ as given by the Lagrangian model. Then $L\left(t, x_{0}\right)=$ $W\left(t, X^{-1}\left(t, x_{0}\right)\right)$, provided that $t$ is a time point when $X^{-1}\left(t, x_{0}\right)$ exists uniquely, i.e. provided that no folding occurs at time $t$. The Lagrangian sea satisfies, by definition, $L(t, X(t, u))=$ $W(t, u)$. By differentiating this relation with respect to $t$, taking (4) into account, the following formula for the temporal derivative is obtained:

$$
L_{t}(t, X(t, u))=W_{t}(t, u)-W_{u}(t, u) \frac{X_{t}(t, u)}{X_{u}(t, u)} .
$$

Again, owing to folding, $L\left(t, x_{0}\right)$ may not be differentiable for certain values of $t$. For such $t$-values, there are multiple $u$ s satisfying $X(t, u)=x_{0}$ and, for each such solution $u$, we define $L_{t}\left(t, x_{0}\right)$ by $(8)$. This should be seen as a purely mathematical construction necessary to get around the problem of the folding of the Lagrangian sea.

The number of $v$-crossings of the Lagrange time wave on the unit interval is given by $N^{*}=\#\{t \in[0,1] ; L(t, 0)=v\}$, where we have set $x_{0}=0$. However, this definition is not convenient to work with when it comes to computing the crossing intensities or the Palm distributions. Instead, note that if $t_{k} \in[0,1]$ is a time when $L(t, 0)=v$ then there exists at least one reference coordinate $u$ satisfying $W\left(t_{k}, u\right)=v$ and $X\left(t_{k}, u\right)=0$. In fact it follows, by direct application of Rice's formula, that there is a.s. only one such $u$. An alternative definition of the number of crossings is thus given by $N=\#\{(t, u) \in[0,1] \times \mathbb{R} ; W(t, u)=v, X(t, u)=0\}$. This crossing definition can easily be extended to include the slope at the crossing. Taking (8) into account, the number of $v$-crossings having slope less than or equal to $y$ is given by

$$
\begin{gathered}
N_{(-\infty, y]}=\#\{(t, u) \in[0,1] \times \mathbb{R} ; W(t, u)=v, X(t, u)=0, \\
\left.W_{t}(t, u)-W_{u}(t, u) \frac{X_{t}(t, u)}{X_{u}(t, u)} \leq y\right\} .
\end{gathered}
$$

Thus, by defining the crossings in this way the problem of computing the crossing intensity and the Palm distribution of slope for the Lagrange time wave is reduced to a two-dimensional crossing problem for Gaussian processes. The solution to this problem is given by the following theorem. 
Theorem 2. (Time waves.) Let $\nabla X=\left(X_{t}(0,0), X_{u}(0,0)\right)^{\top}$ and

$$
\Sigma_{W}=\left(\begin{array}{cc}
r_{u u}^{w w} & -r_{t u}^{w w} \\
-r_{t u}^{w w} & r_{t t}^{w w}
\end{array}\right)
$$

Then we have the following results.

(a) The intensity of $v$-crossings for the Lagrange time wave is given by

$$
\mathrm{E}[N]=\mathrm{E}\left[\sqrt{(\nabla X)^{\top} \boldsymbol{\Sigma}_{W} \nabla X} \mid W(0,0)=v\right] \frac{1}{\pi} \sqrt{\frac{1}{r^{w w}}} \mathrm{e}^{-v^{2} / 2 r^{w w}} .
$$

(b) The (Palm) density of slope at an arbitrary $v$-crossing of the Lagrange time wave is given by

$$
\begin{aligned}
f^{v}(y)=\mathrm{E}[ & \left.\frac{1}{2}|y| \frac{X_{u}^{2}(0,0)}{(\nabla X)^{\top} \boldsymbol{\Sigma}_{W} \nabla X} \exp \left(-\frac{y^{2} X_{u}^{2}(0,0)}{2(\nabla X)^{\top} \boldsymbol{\Sigma}_{W} \nabla X}\right) \sqrt{(\nabla X)^{\top} \boldsymbol{\Sigma}_{W} \nabla X} \mid W(0,0)=v\right] \\
& \times \frac{1}{\mathrm{E}\left[\sqrt{(\nabla X)^{\top} \boldsymbol{\Sigma}_{W} \nabla X} \mid W(0,0)=v\right]} .
\end{aligned}
$$

The proof of Theorem 2 relies on the following lemma, which can be proved by straightforward calculations.

Lemma 3. If $X \in N\left(\mu, \sigma^{2}\right)$ then

$$
\begin{aligned}
& \mathrm{E}\left[|X| \mathbf{1}_{\{X \leq a\}}\right] \\
& = \begin{cases}\mu\left(\Phi\left(\frac{a-\mu}{\sigma}\right)-2 \Phi\left(-\frac{\mu}{\sigma}\right)\right)+\frac{\sigma}{\sqrt{2 \pi}}\left(2 \mathrm{e}^{-\mu^{2} / 2 \sigma^{2}}-\mathrm{e}^{-(a-\mu)^{2} / 2 \sigma^{2}}\right), & a \geq 0, \\
-\mu \Phi\left(\frac{a-\mu}{\sigma}\right)+\frac{\sigma}{\sqrt{2 \pi}} \mathrm{e}^{-(a-\mu)^{2} / 2 \sigma^{2}}, & a<0 .\end{cases}
\end{aligned}
$$

Proof of Theorem 2. The main part of the proof consists of finding an expression for $\mathrm{E}\left[N_{(-\infty, y]}\right]$. Using the version of Rice's formula for marked crossings given in [12] and applying monotone convergence it follows that

$$
\mathrm{E}\left[N_{(-\infty, y]}\right]=\int_{0}^{1} \int_{-\infty}^{\infty} g(t, u) f_{W(t, u), X(t, u)}(v, 0) \mathrm{d} u \mathrm{~d} t,
$$

where, writing $Z(t, u)=(W(t, u), X(t, u))$,

$$
\begin{aligned}
g(t, u)=\mathrm{E}[ & \left|W_{t}(t, u) X_{u}(t, u)-W_{u}(t, u) X_{t}(t, u)\right| \\
& \left.\times \mathbf{1}_{\left\{W_{t}(t, u)-W_{u}(t, u) X_{t}(t, u) / X_{u}(t, u) \leq y\right\}} \mid Z(t, u)=(v, 0)\right] .
\end{aligned}
$$

Using the fact that, conditional on $Z(t, u)=(v, 0)$, the vectors $\left(W_{t}(t, u), W_{u}(t, u)\right)$ and $\left(X_{t}(t, u), X_{u}(t, u)\right)$ are independent (see (13) in Appendix C), the integrand in (11) can be written as

$$
\begin{aligned}
& g(t, u) f_{Z(t, u)}(v, 0)=\int_{-\infty}^{\infty} \int_{-\infty}^{\infty} \mathrm{E}\left[\left|x_{2} W_{t}(t, u)-x_{1} W_{u}(t, u)\right| \mathbf{1}_{A} \mid Z(t, u)=(v, 0)\right] \\
& \times f_{X_{t}, X_{u}, Z}\left(x_{1}, x_{2}, v, 0\right) \mathrm{d} x_{2} \mathrm{~d} x_{1},
\end{aligned}
$$


where $A=\left\{W_{t}(t, u)-W_{u}(t, u) x_{1} / x_{2} \leq y\right\}$ and the variable $(t, u)$ is suppressed in the expression for the density. The distribution of $x_{2} W_{t}(t, u)-x_{1} W_{u}(t, u)$, conditional on $Z(t, u)=$ $(v, 0)$, is normal with mean and variance respectively given by

$$
\begin{gathered}
\mu=\frac{u}{r^{x x}}\left(r_{u 0}^{w x} x_{1}-r_{t 0}^{w x} x_{2}\right), \\
\sigma^{2}=r_{u u}^{w w} x_{1}^{2}+r_{t t}^{w w} x_{2}^{2}-2 r_{t u}^{w w} x_{1} x_{2}-\frac{1}{r^{x x}}\left(r_{u 0}^{w x} x_{1}-r_{t 0}^{w x} x_{2}\right)^{2} ;
\end{gathered}
$$

see Appendix C. Consequently, Lemma 3 can be used to conclude that

$$
\begin{aligned}
& \mathrm{E}\left[\left|x_{2} W_{t}(t, u)-x_{1} W_{u}(t, u)\right| \mathbf{1}_{A} \mid Z(t, u)=(v, 0)\right] \\
& = \begin{cases}\mu\left(\Phi\left(\frac{x_{2} y-\mu}{\sigma}\right)-2 \Phi\left(-\frac{\mu}{\sigma}\right)\right) & \\
\quad+\frac{\sigma}{\sqrt{2 \pi}}\left(2 \exp \left(-\frac{\mu^{2}}{2 \sigma^{2}}\right)-\exp \left(-\frac{\left(x_{2} y-\mu\right)^{2}}{2 \sigma^{2}}\right)\right), & x_{2} \geq 0, y \geq 0, \\
-\mu \Phi\left(\frac{x_{2} y-\mu}{\sigma}\right)+\frac{\sigma}{\sqrt{2 \pi}} \exp \left(-\frac{\left(x_{2} y-\mu\right)^{2}}{2 \sigma^{2}}\right), & x_{2} \geq 0, y<0, \\
\mu\left(\Phi\left(\frac{x_{2} y-\mu}{\sigma}\right)-2 \Phi\left(-\frac{\mu}{\sigma}\right)+1\right) & \\
\quad+\frac{\sigma}{\sqrt{2 \pi}}\left(2 \exp \left(-\frac{\mu^{2}}{2 \sigma^{2}}\right)-\exp \left(-\frac{\left(x_{2} y-\mu\right)^{2}}{2 \sigma^{2}}\right)\right), & x_{2}<0, y \geq 0, \\
\mu\left(1-\Phi\left(\frac{x_{2} y-\mu}{\sigma}\right)\right)+\frac{\sigma}{\sqrt{2 \pi}} \exp \left(-\frac{\left(x_{2} y-\mu\right)^{2}}{2 \sigma^{2}}\right), & x_{2}<0, y<0 .\end{cases}
\end{aligned}
$$

An integral for the intensity $\mathrm{E}\left[N_{(-\infty, y]}\right]$ is thus obtained by combining this result with (11) and (12). According to Fubini's theorem the integration can be carried out in any order. By first integrating with respect to $u$ and using stationarity in the variable $t$, quite lengthy calculations lead to

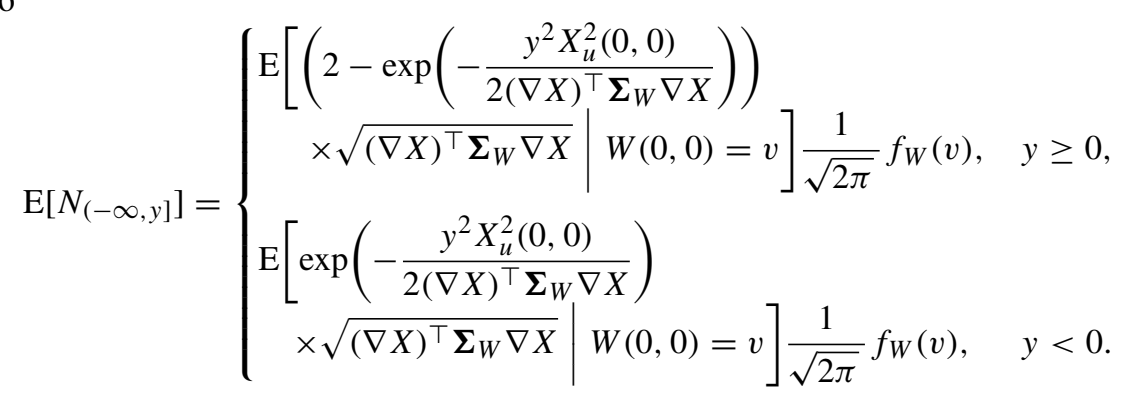

Theorem 2(a) is now obtained by letting $y$ tend to $\infty$ in this expression. Theorem 2(b) follows by forming a Palm distribution by dividing $\mathrm{E}\left[N_{(-\infty, y]}\right]$ by the intensity from Theorem 2 (a), and differentiating with respect to $y$ inside the expectation sign using a dominated convergence argument. This concludes the proof.

Remark 3. The $v$-crossing intensity for $W(t, 0)$, given by Rice's formula, is

$$
\mathrm{E}[\#\{t \in[0,1] ; W(t, 0)=v\}]=\frac{1}{\pi} \sqrt{\frac{r_{t t}^{w w}}{r^{w w}}} \mathrm{e}^{-v^{2} / 2 r^{w w}} .
$$


Comparing this with the crossing intensity, (9), for the Lagrangian sea we see that the $\sqrt{r_{t t}^{w w}}$ factor in the Gaussian case is replaced with an expectation of the square root of a quadratic form. Moreover, in a Gaussian model the slope at an arbitrary crossing has a double Rayleigh distribution, i.e. has a density $\left(1 / 2 r_{t t}^{w w}\right)|r| \mathrm{e}^{-r^{2} / 2 r_{t t}^{w w}}$. A similar factor is present in the Lagrangian expression for slope, given by (10), showing the close relationship between the Lagrangian and Gaussian models. By reducing the Lagrangian model to the Gaussian model, by letting $X(t, u)=u$, both (9) and (10) reduce to their corresponding quantities in the Gaussian model.

Remark 4. With an analogous proof we can show that the (Palm) density of slope at an arbitrary $v$-upcrossing of the Lagrange time wave takes the form

$$
\begin{aligned}
f^{v^{+}}(y)= & \mathrm{E}\left[y \frac{X_{u}^{2}(0,0)}{(\nabla X)^{\top} \boldsymbol{\Sigma}_{W} \nabla X} \exp \left(-\frac{y^{2} X_{u}^{2}(0,0)}{2(\nabla X)^{\top} \boldsymbol{\Sigma}_{W} \nabla X}\right) \sqrt{(\nabla X)^{\top} \boldsymbol{\Sigma}_{W} \nabla X} \mid W(0,0)=v\right] \\
& \times \frac{1}{\mathrm{E}\left[\sqrt{(\nabla X)^{\top} \boldsymbol{\Sigma}_{W} \nabla X} \mid W(0,0)=v\right]}, \quad y>0 .
\end{aligned}
$$

The intensity of $v$-crossings, (9), and the density of slope, (10), of the Lagrange time wave are not given in an explicit form. However, since they are given in terms of expectations of functions of normal variables, it is convenient to evaluate them by simulation or numerical integration. The former approach is used in the example presented next.
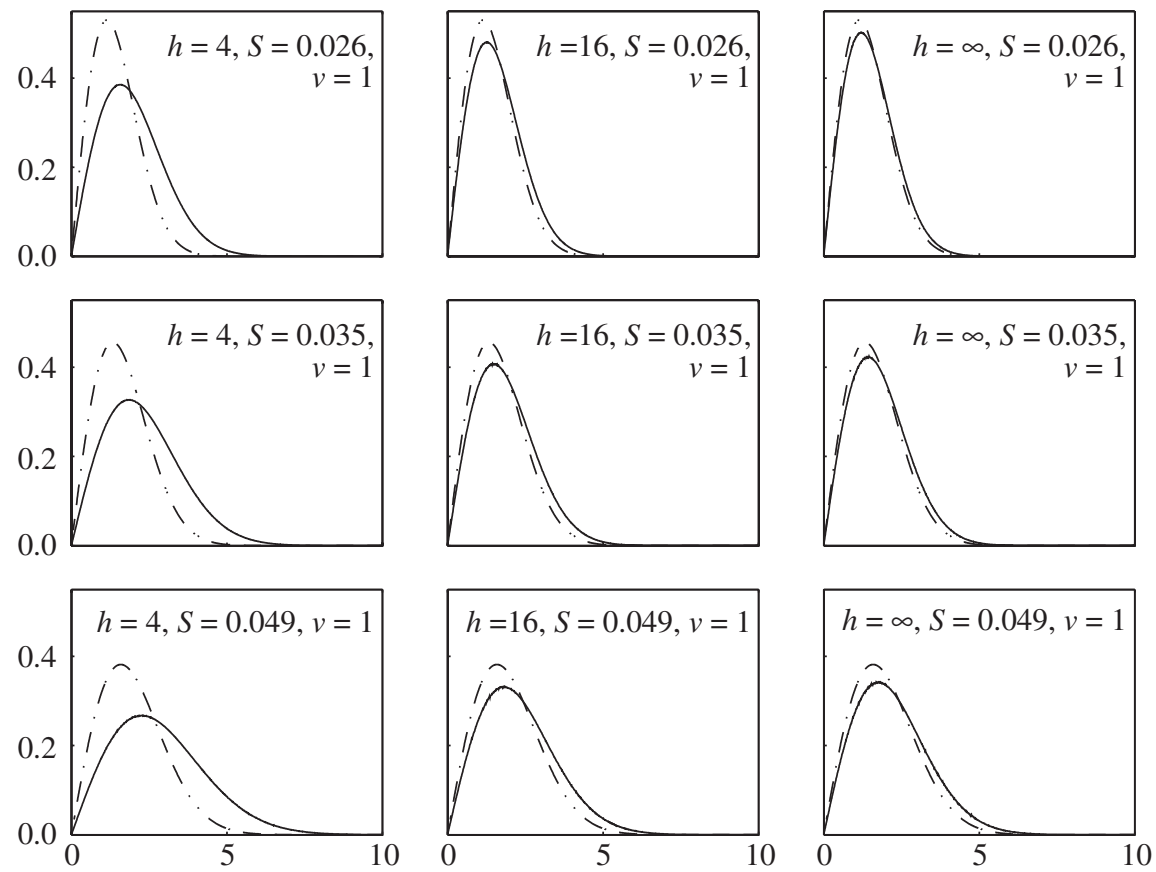

FIGURE 2: Density of slope observed at an arbitrary time-wave upcrossing of level $v=1 \mathrm{~m}$ in the Gaussian (dash-dotted line) and Lagrangian (solid line) models for different water depths, $h=4,16$, and $\infty \mathrm{m}$, and spectrum steepnesses, $S=0.026,0.035$, and 0.049 . 

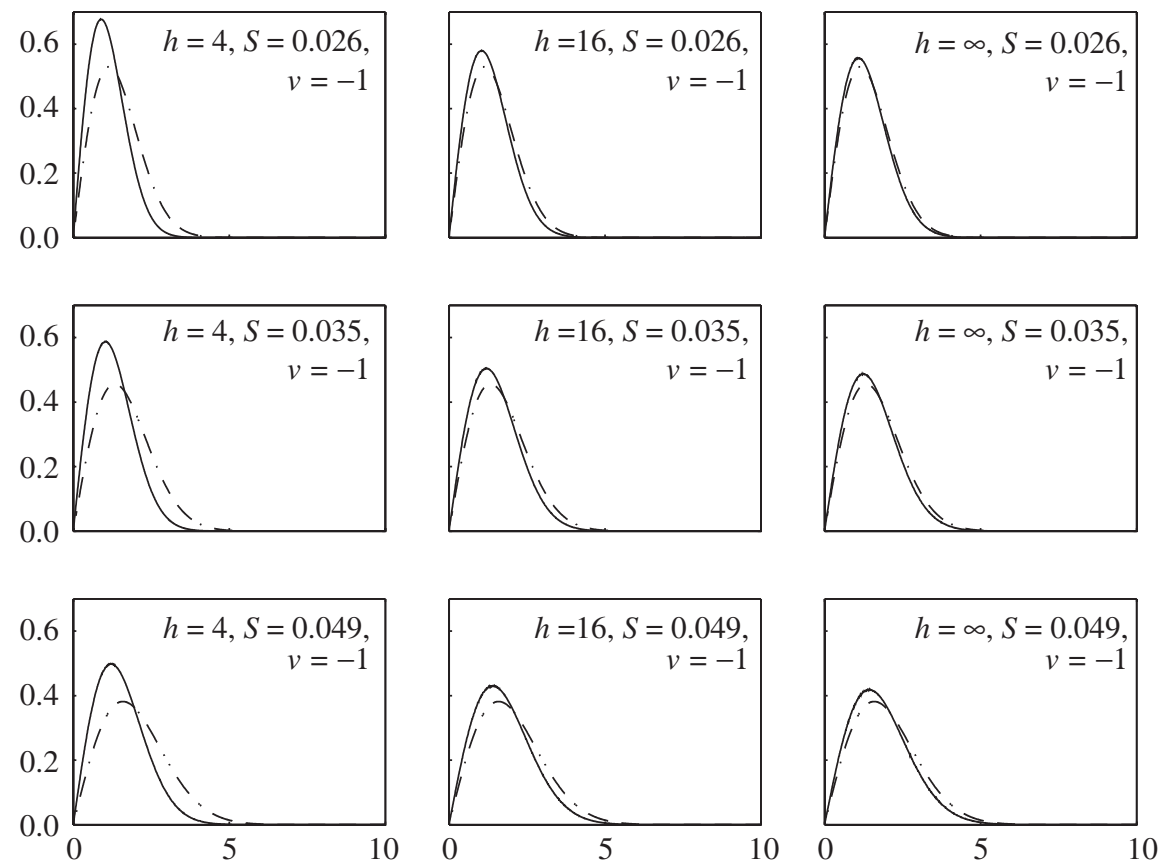

FIGURE 3: Density of slope observed at an arbitrary time-wave upcrossing of level $v=-1 \mathrm{~m}$ in the Gaussian (dash-dotted line) and Lagrangian (solid line) models for different water depths, $h=4,16$, and $\infty \mathrm{m}$, and spectrum steepnesses, $S=0.026,0.035$, and 0.049 .

Example 2. The purpose of this example is to show the differences between the densities of slope at time-wave upcrossings for the Gaussian and the Lagrangian models. Again we consider three Pierson-Moskowitz spectra, each having different spectrum steepnesses and different water depths. The same parameters as in Example 1 are chosen, i.e. water depths of 4,16 , and $\infty \mathrm{m}$, spectrum steepnesses of $S=0.026,0.035$, and 0.049 , and a significant wave height of $H_{\mathrm{s}}=7 \mathrm{~m}$. Based on a sample size of 1000, the density for the Lagrangian model is evaluated by simulation and the resulting curves are shown in Figures 2 and 3. Figures 2 and 3 show the density of slope for the Lagrange time wave for crossing levels of $v=1 \mathrm{~m}$ and $v=-1 \mathrm{~m}$, respectively. From these plots it can be seen that the Lagrange densities are dependent on the crossing level, whereas the densities arising from the Gaussian model are not. Furthermore, the same pattern is present in these figures as in the corresponding space-wave densities in Example 1, namely, that the difference between the models increases with steepness and decreases with water depth.

\section{Appendix A. Spectrum and covariances}

By choosing $\kappa$ and $\omega$ to have the same sign in the spectral representation, (1), the waves are traveling in the direction of the positive $x$-axis and if they have different signs then the waves are traveling in the opposite direction. In order to have a model where both wave directions are allowed we may add two independent processes, one in which the waves move to the right and 
one in which the waves move to the left, i.e.

$$
W(t, u)=\int_{-\infty}^{\infty} \mathrm{e}^{\mathrm{i}\left(\kappa^{+}(\omega) u-\omega t\right)} \mathrm{d} \xi^{+}(\omega)+\int_{-\infty}^{\infty} \mathrm{e}^{\mathrm{i}\left(\kappa^{-}(\omega) u-\omega t\right)} \mathrm{d} \xi^{-}(\omega) .
$$

Here $\kappa^{+}(\omega)$ denotes that $\kappa$ is chosen to have the same sign as $\omega$ and $\kappa^{-}(\omega)$ denotes that $\kappa$ and $\omega$ are of opposite sign. Each of the spectral processes $\xi^{+}(\omega)$ and $\xi^{-}(\omega)$, which are assumed to be independent, can be related to a spectral density, $\tilde{S}^{+}(\omega)$ and $\tilde{S}^{-}(\omega)$, say. The spectral density $\tilde{S}^{+}(\omega)$ is defined by the relation

$$
\mathrm{E}\left[\mathrm{d} \xi^{+}(\omega) \overline{\mathrm{d} \xi^{+}\left(\omega^{\prime}\right)}\right]= \begin{cases}0, & \omega \neq \omega^{\prime} \\ \tilde{S}^{+}(\omega) \mathrm{d} \omega, & \omega=\omega^{\prime}\end{cases}
$$

with $\tilde{S}^{-}(\omega)$ defined in an analogous way. Moreover, the symmetry relations $\mathrm{d} \xi^{+}(-\omega)=$ $\overline{\mathrm{d} \xi^{+}(\omega)}$ and $\mathrm{d} \xi^{-}(-\omega)=\overline{\mathrm{d} \xi^{-}(\omega)}$, which ensure that the process $W(t, u)$ is real valued, imply that $\tilde{S}^{+}(\omega)$ and $\tilde{S}^{-}(\omega)$ are symmetric. In oceanography, however, we do not work with the spectra $\tilde{S}^{+}(\omega)$ and $\tilde{S}^{-}(\omega)$, but rather with a frequency spectrum $S(\omega)$, say, which is asymmetric and constructed in such a way that positive frequencies indicate that the wave is traveling to the right and negative frequencies indicate that the wave is traveling to the left. The relation between the different spectra is given by

$$
S(\omega)= \begin{cases}2 \tilde{S}^{+}(\omega), & \omega>0, \\ \tilde{S}^{+}(0)+\tilde{S}^{-}(0), & \omega=0 \\ 2 \tilde{S}^{-}(\omega), & \omega<0 .\end{cases}
$$

The interpretation of the spectral density for the Gaussian model is quite different from that for the Lagrangian model. In the Gaussian model the spectrum describes the distribution of frequencies of the sea surface at a fixed point, whereas in the Lagrangian model it describes a distribution of frequencies in the elevation of a fixed particle $u$, which is clearly nonfixed since it moves according to $X(t, u)$. Owing to this fact the spectrum in the Lagrangian model is called an orbital spectrum. As noted in [5], few studies have been carried out on suitable models for the orbital spectrum, and in our case we use the same input spectrum as for the Gaussian model.

Below, covariances and cross-covariances are given for the processes $X(t, u)$ and $W(t, u)$ in the Lagrangian model. These formulae are corrected versions of the ones that can be found in [9]. In the following $S(\omega)$ is the asymmetric frequency spectrum and notation such as $r_{u 0}^{w x}(t, u)=\operatorname{cov}\left(W_{u}(0,0), X(t, u)\right)$ is used for the different covariances.

The covariances of $W(t, u)$ and its derivatives are given by

$$
\begin{aligned}
& r^{w w}(t, u)=\int_{-\infty}^{\infty} \cos (|\kappa| u-\omega t) S(\omega) \mathrm{d} \omega, \\
& r_{t 0}^{w w}(t, u)=-\int_{-\infty}^{\infty} \omega \sin (|\kappa| u-\omega t) S(\omega) \mathrm{d} \omega, \\
& r_{u 0}^{w w}(t, u)=\int_{-\infty}^{\infty}|\kappa| \sin (|\kappa| u-\omega t) S(\omega) \mathrm{d} \omega, \\
& r_{t u}^{w w}(t, u)=-\int_{-\infty}^{\infty} \omega|\kappa| \cos (|\kappa| u-\omega t) S(\omega) \mathrm{d} \omega,
\end{aligned}
$$




$$
\begin{aligned}
& r_{t t}^{w w}(t, u)=\int_{-\infty}^{\infty} \omega^{2} \cos (|\kappa| u-\omega t) S(\omega) \mathrm{d} \omega, \\
& r_{u u}^{w w}(t, u)=\int_{-\infty}^{\infty}|\kappa|^{2} \cos (|\kappa| u-\omega t) S(\omega) \mathrm{d} \omega .
\end{aligned}
$$

The covariances of $X(t, u)$ and its derivatives are given by

$$
\begin{aligned}
& r^{x x}(t, u)=\int_{-\infty}^{\infty} \cos (|\kappa| u-\omega t)\left(\frac{\cosh (|\kappa| h)}{\sinh (|\kappa| h)}\right)^{2} S(\omega) \mathrm{d} \omega, \\
& r_{t 0}^{x x}(t, u)=-\int_{-\infty}^{\infty} \omega \sin (|\kappa| u-\omega t)\left(\frac{\cosh (|\kappa| h)}{\sinh (|\kappa| h)}\right)^{2} S(\omega) \mathrm{d} \omega, \\
& r_{u 0}^{x x}(t, u)=\int_{-\infty}^{\infty}|\kappa| \sin (|\kappa| u-\omega t)\left(\frac{\cosh (|\kappa| h)}{\sinh (|\kappa| h)}\right)^{2} S(\omega) \mathrm{d} \omega, \\
& r_{t u}^{x x}(t, u)=-\int_{-\infty}^{\infty} \omega|\kappa| \cos (|\kappa| u-\omega t)\left(\frac{\cosh (|\kappa| h)}{\sinh (|\kappa| h)}\right)^{2} S(\omega) \mathrm{d} \omega, \\
& r_{t t}^{x x}(t, u)=\int_{-\infty}^{\infty} \omega^{2} \cos (|\kappa| u-\omega t)\left(\frac{\cosh (|\kappa| h)}{\sinh (|\kappa| h)}\right)^{2} S(\omega) \mathrm{d} \omega, \\
& r_{u u}^{x x}(t, u)=\int_{-\infty}^{\infty}|\kappa|^{2} \cos (|\kappa| u-\omega t)\left(\frac{\cosh (|\kappa| h)}{\sinh (|\kappa| h)}\right)^{2} S(\omega) \mathrm{d} \omega .
\end{aligned}
$$

The cross-covariances of $W(t, u)$ and $X(t, u)$ and their derivatives are given by

$$
\begin{aligned}
& r^{w x}(t, u)=-\int_{-\infty}^{\infty} \sin (|\kappa| u-\omega t) \frac{\cosh (|\kappa| h)}{\sinh (|\kappa| h)} S(\omega) \mathrm{d} \omega, \\
& r_{u 0}^{w x}(t, u)=\int_{-\infty}^{\infty}|\kappa| \cos (|\kappa| u-\omega t) \frac{\cosh (|\kappa| h)}{\sinh (|\kappa| h)} S(\omega) \mathrm{d} \omega, \\
& r_{t 0}^{w x}(t, u)=-\int_{-\infty}^{\infty} \omega \cos (|\kappa| u-\omega t) \frac{\cosh (|\kappa| h)}{\sinh (|\kappa| h)} S(\omega) \mathrm{d} \omega, \\
& r_{t u}^{w x}(t, u)=r_{u t}^{w x}(t, u)=\int_{-\infty}^{\infty} \omega|\kappa| \sin (|\kappa| u-\omega t) \frac{\cosh (|\kappa| h)}{\sinh (|\kappa| h)} S(\omega) \mathrm{d} \omega, \\
& r_{t t}^{w x}(t, u)=-\int_{-\infty}^{\infty} \omega^{2} \sin (|\kappa| u-\omega t) \frac{\cosh (|\kappa| h)}{\sinh (|\kappa| h)} S(\omega) \mathrm{d} \omega, \\
& r_{u u}^{w x}(t, u)=-\int_{-\infty}^{\infty}|\kappa|^{2} \sin (|\kappa| u-\omega t) \frac{\cosh (|\kappa| h)}{\sinh (|\kappa| h)} S(\omega) \mathrm{d} \omega, \\
& r_{u 0}^{x w}(t, u)=-r_{u 0}^{w x}(t, u), \\
& r_{t 0}^{x w}(t, u)=-r_{t 0}^{w x}(t, u) .
\end{aligned}
$$

\section{Appendix B. The Pierson-Moskowitz spectrum}

The Pierson-Moskowitz (PM) spectrum is a standard spectrum in ocean sciences and is used to model a fully developed sea. The concept of a fully developed sea means that it has blown steadily for a long time over a large area so that the waves are in equilibrium with the wind. The PM spectrum has the following parametric form:

$$
S(\omega)=\frac{5 H_{\mathrm{s}}^{2}}{\omega_{\mathrm{p}}\left(\omega / \omega_{\mathrm{p}}\right)^{5}} \exp \left(-\frac{5}{4} \frac{1}{\left(\omega / \omega_{\mathrm{p}}\right)^{4}}\right),
$$


where $H_{\mathrm{s}}$ is the significant wave height, defined as four times the standard deviation of the surface elevation, and $\omega_{\mathrm{p}}$ is the peak frequency, i.e. the frequency where the spectrum has its peak. Sometimes the peak period $T_{\mathrm{p}}$, defined by $T_{\mathrm{p}}=2 \pi / \omega_{\mathrm{p}}$, is used instead of the peak period $\omega_{\mathrm{p}}$. Thus, the PM spectrum is totally defined by the two parameters $H_{\mathrm{s}}$ and $T_{\mathrm{p}}$. Often, however, we are mainly interested in the large structures of the sea surface and not in the fine ripples on the surface, represented by the high frequencies in the spectrum. In this case the spectrum is 'cut off' at a certain frequency $\omega_{\mathrm{c}}$, called the cut-off frequency, meaning that $S(\omega)$ is set to 0 for all $\omega>\omega_{\mathrm{c}}$.

\section{Appendix C. Distributions}

In this section distributions are given which are necessary to evaluate the crossing intensities and the Palm distributions of slope for the Lagrangian sea. In the following we use the notation $r_{t t}^{w w}=r_{t t}^{w w}(0,0)$, etc.

Let $\boldsymbol{Y}=\left(W_{t}(t, u), W_{u}(t, u), X_{t}(t, u), X_{u}(t, u)\right)^{\top}$ and $\boldsymbol{Z}=(W(t, u), X(t, u))^{\top}$. Then the joint distribution of $\left(\boldsymbol{Y}^{\top}, \boldsymbol{Z}^{\top}\right)^{\top}$ is normal with expectation $\boldsymbol{\mu}$ and covariance $\boldsymbol{\Sigma}$ given by

$$
\boldsymbol{\mu}=\left(\begin{array}{l}
0 \\
0 \\
0 \\
1 \\
0 \\
u
\end{array}\right), \quad \boldsymbol{\Sigma}=\left(\begin{array}{cccccc}
r_{t t}^{w w} & r_{t u}^{w w} & 0 & 0 & 0 & r_{t 0}^{w x} \\
r_{t u}^{w w} & r_{u u}^{w w} & 0 & 0 & 0 & r_{u 0}^{w x} \\
0 & 0 & r_{t t}^{x x} & r_{t u}^{x x} & r_{t 0}^{x w} & 0 \\
0 & 0 & r_{t u}^{x x} & r_{u u}^{x x} & r_{u 0}^{x w} & 0 \\
0 & 0 & r_{t 0}^{x w} & r_{u 0}^{x w} & r^{w w} & 0 \\
r_{t 0}^{w x} & r_{u 0}^{w x} & 0 & 0 & 0 & r^{x x}
\end{array}\right)
$$

Since the joint distribution is normal then the conditional distribution of $\boldsymbol{Y}$, given that $\boldsymbol{Z}=$ $(v, 0)^{\top}$, is also normal. Denoting the conditional mean and the covariance by $\boldsymbol{\mu}_{\boldsymbol{Y} \mid \boldsymbol{Z}=(v, 0)^{\top}}$ and $\boldsymbol{\Sigma}_{\boldsymbol{Y} \mid \boldsymbol{Z}=(v, 0)^{\top}}$, respectively, we have

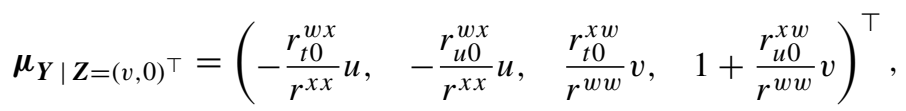

$$
\begin{aligned}
& \boldsymbol{\Sigma}_{\boldsymbol{Y} \mid \boldsymbol{Z}=(v, 0)^{\top}}=\left(\begin{array}{cccc}
r_{t t}^{w w}-\frac{\left(r_{t 0}^{w x}\right)^{2}}{r^{x x}} & r_{t u}^{w w}-\frac{r_{t 0}^{w x} r_{u 0}^{w x}}{r^{x x}} & 0 & 0 \\
r_{t u}^{w w}-\frac{r_{t 0}^{w x} r_{u 0}^{w x}}{r^{x x}} & r_{u u}^{w w}-\frac{\left(r_{u 0}^{w x}\right)^{2}}{r^{x x}} & 0 & 0 \\
0 & 0 & r_{t t}^{x x}-\frac{\left(r_{t 0}^{x w}\right)^{2}}{r^{w w}} & r_{t u}^{x x}-\frac{r_{u 0}^{x w} r_{t 0}^{x w}}{r^{w w}} \\
0 & 0 & r_{t u}^{x x}-\frac{r_{u 0}^{x w} r_{t 0}^{x w}}{r^{w w}} & r_{u u}^{x x}-\frac{\left(r_{u 0}^{x w}\right)^{2}}{r^{w w}}
\end{array}\right) .
\end{aligned}
$$

Note that, conditional on $\boldsymbol{Z}=(v, 0)^{\top}$, the pairs $\left(W_{t}(t, u), W_{u}(t, u)\right)$ and $\left(X_{t}(t, u), X_{u}(t, u)\right)$ are independent.

\section{Acknowledgement}

The author is grateful to an anonymous referee whose comments led to a significant improvement of the paper. 


\section{References}

[1] Åberg, S., Rychlik, I. and Leadbetter, M. R. (2006). Palm distributions of wave characteristics in encountering seas. To appear in Ann. Appl. Prob.

[2] Azaïs, J. M., León, J. R. And ORtega, J. (2005). Geometrical characteristics of Gaussian sea waves. J. Appl. Prob. 42, 407-425.

[3] Baxevani, A., Podgórski, K. and Rychlik, I. (2003). Velocities for moving random surfaces. Prob. Eng. Mech. 18, 251-271.

[4] Cramér, H. and Leadbetter, M. R. (1967). Stationary and Related Stochastic Processes. John Wiley, New York.

[5] Fouques, S., Krogstad, H. E. and Myrhaug, D. (2006). A second order Lagrangian model for irregular ocean waves. Trans. AMSE J. Offshore Mech. Arctic Eng. 128, 177-183.

[6] GJøsund, S. H. (2003). A Lagrangian model for irregular waves and wave kinematics. Trans. AMSE J. Offshore Mech. Arctic Eng. 125, 94-102.

[7] Lagrange, J. L. (1788). Mécanique Analytique. Jacques Gabay, Paris.

[8] Leadbetter, M. R., Lindgren, G. And Rootzén, H. (1983). Extremes and Related Properties of Random Sequences and Processes. Springer, New York.

[9] Lindgren, G. (2006). Slepian models for the stochastic shape of individual Lagrange sea waves. Adv. Appl. Prob. 38, 430-450.

[10] LindgREN, G. AND RYCHLIK, I. (1991). Slepian models and regression approximation in crossings and extreme value theory. Internat. Statist. Rev. 59, 195-225.

[11] Longuet-Higgins, M. S. (1957). The statistical analysis of a random, moving surface. Phil. Trans. R. Soc. London A 249, 321-387.

[12] Mercadier, C. (2006). Numerical bounds for the distributions of the maxima of some one- and two-parameter Gaussian processes. Adv. Appl. Prob. 38, 149-170

[13] Podgórski, K., Rychlik, I. And Machado, U. E. B. (2000). Exact distributions for apparent waves in irregular seas. Ocean Eng. 27, 979-1016.

[14] Socquet-Juglard, H. et al. (2004). Spatial extremes, shapes of large waves, and Lagrangian models. In Proc. Rogue Waves (Brest, 2004). IFREMER, Plouzané. Available at http://ifremer.fr/web-com/ stw2004/rw/fullpapers/krogstad.pdf.

[15] St. Denis, M. and Pierson, W. J. (1953). On the motion of ships in confused seas. Trans. Soc. Naval Architects Marine Eng. 61, 280-357. 